\title{
Development of kinetic parameters for polyurethane thermal degradation modeling featuring a bioinspired catecholic flame retardant
}

\author{
B.C. Roberts ${ }^{\mathrm{a}, *}$, A.R. Jones ${ }^{\mathrm{b}}$, O.A. Ezekoye ${ }^{\mathrm{a}}$, C.J. Ellison ${ }^{\mathrm{b}}$, M.E. Webber ${ }^{\mathrm{a}}$ \\ ${ }^{a}$ The University of Texas at Austin, Department of Mechanical Engineering, Cockrell \\ School of Engineering, 204 E. Dean Keeton St. C2200, Austin, TX 78712 \\ ${ }^{b}$ The University of Texas at Austin, Department of Chemical Engineering, Cockrell \\ School of Engineering, 1 University Station C0400, Austin, TX 78712
}

\section{Abstract}

A recently developed flame retardant (FR) nanocoating of polydopamine (PDA) was applied to flexible polyurethane foam (PU) and thermogravimetrically analyzed (TGA). Thermal degradation kinetics were described by a simplified multi-component, Arrhenius expression coupled with a first-order reaction model. Kinetic parameters were then extracted via an optimization solver. By limiting the number of optimized parameters, a mesh adaptive direct search algorithm was employed to extract meaningful kinetic parameters that better simulate the TGA data compared to graphical methods. Through TGA, it was shown that the effect of the PDA nanocoating on PU degradation differs between oxidative (78 vol\% nitrogen $\left(\mathrm{N}_{2}\right)$ and 21 vol\% oxygen) and inert $\left(100 \% \mathrm{~N}_{2}\right)$ environments. In nitrogen, the mass loss is delayed and diminished in the first PU reaction, which is the opposite effect

${ }^{*}$ Corresponding author. +15124756867 .

Email addresses: broberts@utexas.edu (B.C. Roberts), ajones@che.utexas.edu (A.R. Jones), dezekoye@mail.utexas.edu (O.A. Ezekoye), ellison@che.utexas.edu (C.J. Ellison), webber@mail.utexas.edu (M.E. Webber) 
of a traditional halophosphate FR. In an oxidative environment, the first reaction of $\mathrm{PU}$ is greatly delayed by the PDA coating, but once the reaction begins, it becomes accelerated.

Keywords: Flame Retardant, Polydopamine, Flexible Polyurethane Foam, Thermal Degradation, Kinetic Parameters, Thermogravimetric Analysis

\section{Introduction}

Used in a variety of cushioning applications, including mattresses, upholstered furniture, and carpeting, flexible polyurethane foam (PU) has been in high demand since becoming commercially available in the early 1950s. $\mathrm{PU}$ is the product of a reaction between a reactive hydrogen containing compound and a polyisocyanate. Typical formulations involve a polyol reacted with toluene diisocyanate (TDI) [1]. According to the Polyurethane Foam Association (PFA), over 1.2 billion pounds (544,311 metric tons) of PU are produced in the U.S. annually [2]. Although PU has many benefits, it is a highly flammable material that, depending on the application, can require additional protection to meet U.S. fire codes and standards. Therefore, flame retardants (FRs) are typically incorporated to meet flammability standards. According to a 2015 report by the U.S. Environmental Protection Agency (EPA), there are very few FR options for PU that do not pose a high health concern for humans, aquatic life, and the environment [3]. Although halogenated FRs have been criticized for their toxicity, the EPA indicates that many non-halogenated phosphate alternatives still pose very high aquatic

toxicity hazards along with moderate to high human health concerns [3]. Due to the limited availability of nontoxic, environmentally friendly FR op- 
tions, research and development of new FRs is still needed.

The FR investigated in this study is polydopamine (PDA), which is a synthetic analog of naturally occurring melanins. Melanins are polyphenolic compounds derived from the oxidation of tyrosine or dihydroxyphenylalanine (DOPA), and are produced by living organisms [4]. In 2007, it was discovered that PDA forms as a protective nanocoating on a number of different materials if dopamine hydrochloride is placed in a slightly basic $(\mathrm{pH}=8.5)$ aqueous solution [4]. Since the discovery, PDA has been studied as a beneficial coating in a variety of applications, such as protective light absorption and drug delivery [4]. Recently, a U.S. patent presented the composition of PDA and either tris(hydroxymethyl)aminomethane or gaseous ammonia as a flame retardant coating [5]. Subsequently, PDA was successfully employed as a surface coating to PU for the purpose of decreasing PU flammability [6]. In the study by Cho et al. [6], microscale combustion calorimetry (MCC) and thermogravimetric analysis (TGA) of neat PDA in nitrogen demonstrated that it is relatively inflammable and forms thermally protective carbonaceous, porous char. Additionally, PDA was deposited on PU after various coating times and the resultant coating was characterized. A significant reduction (67\%) in peak heat release rate was demonstrated using cone calorimetry for foam specimens with 15.9 wt\% PDA deposited, which was achieved after three days of coating. This success was attributed to synergistic combinations of physical and chemical actions, which we explore further in the present work through TGA and thermal degradation modeling in both oxidative and inert environments.

A primary step in understanding and quantifying the effects of FRs is 
the investigation of the impact on thermal degradation. Thermogravimetric analysis is a convenient method of observing changes in physical and chemical properties of materials as a function of a constant heating rate. Only a small sample is required, enabling economical early stage validation of $\mathrm{FR}$ viability in new chemical combinations. Sample mass and temperature are monitored throughout heating, which makes observations of condensed-phase changes possible. Solid-state reaction rate models can then be applied to the empirical data to extract kinetic parameters. In theory, these parameters have physical meaning that can describe the mass loss due to chemical reactions. However, an appropriate kinetic model must be applied [7]. One benefit of extracting kinetic parameters is that they aid complex modeling of ignition and combustion of materials $[8,9,10,11,12,13]$.

Previous studies have investigated pyrolysis and/or oxidation of PU with and without FR via TGA and applied their results to an $n$-order reaction model, similar to the one suggested herein. Consensus among researchers has indicated that an $n$-order model is most appropriate for $\mathrm{PU}$ decomposition reactions, although Rogers and Ohlemiller [14] assessed that applying a twodimensional diffusion model to the first reaction and a nucleation model to the second reaction was best. Chao and Wang [15] proposed thermal degradation mechanisms in both air and nitrogen environments of neat and brominated phosphorus FR PU. Kinetic parameters were graphically determined using the Friedman [16] method. Bilbao et al. [17] used both isothermal and dynamic TGA data to derive kinetic parameters in both inert and oxidative environments for neat PU. A variation of the Arrhenius Plot graphical method was used to extract kinetic parameters. Pau et al. [18] analyzed 
both neat and chlorinated phosphorous melamine FR PU in a nitrogen environment at multiple heating rates. Three different graphical techniques (Kinetic Analysis, Arrhenius Plot, and Inflection Point) were tested to determine their compatibility with built-in pyrolysis models in Fire Dynamics Simulator (FDS) and Gpyro [18, 19, 20]. Prasad et al. [11] simulated TGA, cone calorimeter, and flame spread experiments using FDS after optimizing for kinetic parameters via the built-in genetic algorithm (GA) of Gpyro. Rein et al. $[12,13]$ implemented numerical models for TGA and one-dimensional smoldering combustion under normal and microgravity conditions. Kinetic parameters were estimated using a GA toolbox, GAOT. Garrido and Font [21] extensively studied chlorinated phosphorus FR PU at three different heating rates and environments, with both dynamic and isothermal runs. A proposed degradation model was optimized with the Excel Solver suite. Furthermore, evolution of gaseous compounds was observed with mass spectrometry and Fourier transformed infrared spectroscopy in both inert and oxidative environments [21]. Details on the methods and models used can be found in the Supplementary Material along with a complete list of reported kinetic parameters.

In this study, a theoretical model describing the decomposition kinetics is used to simulate the results of the TGA. Ultimately, the simulation is compared to the empirical data and optimized to find suitable kinetic parameters. These optimized kinetic parameters are compared with literature to confirm the veracity of the model. It is demonstrated that, without the benefit of optimization, graphical methods are not able to effectively describe the mass loss curve. Additionally, discussed in some detail is the indication that PDA 
plays an active role in preventing PU degradation. Analysis is performed by subtracting a hypothetical composite of PDA and PU from actual TGA data. To the author's knowledge, this method of comparison has not been applied to TGA data before. Furthermore, no published studies have examined the kinetics of PDA flame retardancy, nor critically analyzed TGA results in both oxidative and inert environments. Finally, this study indicates that PDA has a different mode of action than traditional halophosphate FRs.

\section{Experimental Procedure}

\subsection{Materials}

Polyether-based flexible polyurethane foam (type 1850, Future Foams, High Point, NC) without flame retardant additives (density of $28 \mathrm{~kg} / \mathrm{m}^{3}$ ) was generously provided and used as received. Dopamine hydrochloride was purchased from Sigma-Aldrich. Tris (tris(hydroxymethyl)aminomethane) base was obtained from Fisher Scientific. Ultrapure water $(18.2 \mathrm{M} \Omega \mathrm{cm})$ was obtained from a Thermo Scientific Barnstead E-pure water purification system.

\subsection{Sample Preparation}

Allowing three days for PDA deposition, the polydopamine coated flexible polyurethane foam (PDA3D) was prepared as described previously by Cho et al. [6]. In brief, the PU was submerged in a solution of dopamine hydrochloride and $10 \mathrm{mM}$ Tris-HCl buffer solution $(2 \mathrm{mg} / \mathrm{ml}$ dopamine: buffer, $\mathrm{pH}$ 8.5) under continuous agitation for three days. With time, the dopamine polymerized and deposited throughout the foam. Specimens were then rinsed with deionized water, dried in air for two days, dried under vacuum for one 
day, and then finally dried under vacuum at $70{ }^{\circ} \mathrm{C}$ to remove residual water and unbound PDA. The percentage of PDA deposited was 15.9 wt\%, as calculated from the initial and final weight of the specimen after coating. It should be noted that the weight of a control foam decreased when immersed in the buffer solution because of leached unattached molecules. The weight of the control foam in the buffer reached steady state after one day, with a total weight loss of about 4.8 wt \%. This weight loss was taken into account in the calculation of the mass of PDA deposited.

\subsection{Thermogravimetric Analysis}

A small ( $\sim 5 \mathrm{mg})$ amount of open-cell, flexible polyurethane foam, with and without PDA coating, denoted as PDA3D and PU, respectively, was heated from $30{ }^{\circ} \mathrm{C}$ to $800{ }^{\circ} \mathrm{C}$ using a thermogravimetric analyzer (DSC/TGA 1, Mettler Toledo). Both an oxidative (ultra-pure air, 78 vol\% nitrogen $\left(\mathrm{N}_{2}\right)$ and 21 vol\% oxygen $\left.\left(\mathrm{O}_{2}\right)\right)$ and inert $\left(100 \% \mathrm{~N}_{2}\right)$ environment were tested at heating rates of 10 and $20^{\circ} \mathrm{C} / \mathrm{min}$ for $\mathrm{PU}$ and PDA3D. The air and nitrogen flows through the TGA were maintained at $50 \mathrm{~mL} / \mathrm{min}$ throughout testing.

\section{Model Description}

A number of reasonable assumptions were made to simplify modeling of the thermal decomposition of polyurethane. For a sufficiently small sample size, the time scale for kinetics is assumed to be long compared to the time scales for heat and mass transfer. The material's mass loss rate (MLR) is not spatially dependent; uniform temperature is assumed throughout the specimen due to the small sample size and relatively low heating rate (i.e. lumped capacitance model). It is further assumed that the chemical reactions 
are not diffusion controlled; there are no porosity effects and gas species are instantaneously released from the solid. Finally, local thermal equilibrium between the solid and gaseous components exists with no condensation of gaseous products.

An Arrhenius expression coupled with reaction models (Eq. 1) was used to describe the thermal decomposition kinetics of the material.

$$
\frac{d \alpha}{d t}=A e^{\frac{-E}{R T}} Y_{O_{2}}{ }^{n O_{2}} f(\alpha)
$$

In Equation 1, conversion, or mass fraction decomposed, is $\alpha=\left(m_{0}-\right.$ $m) /\left(m_{0}-m_{f}\right)$, where $m_{0}, m_{f}$, and $m$ are initial, final, and instantaneous mass, respectively. Additional symbols are time $(t)[\mathrm{s}]$, activation energy $(E)\left[\mathrm{kJ}-\mathrm{mol}^{-1}\right]$, pre-exponential factor $(A)\left[\mathrm{s}^{-1}\right]$, universal gas constant $(R)$ $[\mathrm{J} / \mathrm{mol}-\mathrm{K}]$, temperature $(T)[\mathrm{K}]$, and reaction model $(f(\alpha))$. The atmospheric oxygen mass fraction $\left(Y_{\mathrm{O}_{2}}\right)$ was assumed to be zero for $100 \%$ nitrogen and 0.23 for an air environment. A zero-order reaction $\left(n_{\mathrm{O}_{2}}=0\right)$ was applied in nitrogen and a first-order reaction $\left(n_{O_{2}}=1\right)$ was assumed in air. The information provided from the TGA is not sufficient to derive the reaction order for oxygen. Therefore, a first-order reaction has been assumed, which was also applied by Rein et al. [13].

Different reaction models exist (nucleation, diffusion, etc.), but a phase boundary, a.k.a. $n$-order model (e.g., $\left.f(\alpha)=(1-\alpha)^{n}\right)$, is most frequently used to describe polyurethane decomposition. Assuming an $n$-order reaction model, the rate equation can be rewritten in terms of mass fraction $\left(Y=m / m_{0}\right)$, temperature, and heating rate $(\beta=d T / d t)$, as shown in Equa- 
tion 2. At the end of heating, the final residual mass fraction is $Y_{f}=m_{f} / m_{0}$.

$$
\frac{d Y}{d T}=-\frac{A}{\beta} Y_{O_{2}}^{n_{O_{2}}}\left(Y-Y_{f}\right)^{n}\left(1-Y_{f}\right)^{1-n} e^{\frac{-E}{R T}}
$$

For a material that undergoes multiple reactions while heated at rate $\beta$, the material can be modeled as a composite of $N$ components, each of which undergoes a single reaction. As the material decomposes, each component can contribute to the residual mass fraction $\left(Y_{r}\right)$. Assuming each reaction is first-order $(n=1)$, the state equations describing the material decomposition can be written as:

$$
\begin{gathered}
\frac{d Y_{i}}{d T}=-\frac{A_{i}}{\beta} Y_{i, O_{2}}^{n_{i, O_{2}}} Y_{i} e^{\frac{-E_{i}}{R T}} \quad ; i=1, . . N \\
\frac{d Y_{r}}{d T}=-Y_{f} \sum_{i=1}^{N} \frac{d Y_{i}}{d T} \\
\frac{d Y}{d T}=\sum_{i=1}^{N} \frac{d Y_{i}}{d T}+\frac{d Y_{r}}{d T}
\end{gathered}
$$

Equation 5 is necessary for establishing the interdependency of the total mass fraction $(Y)$ of the material to the individual component mass fractions $\left(Y_{i}\right)$. This necessity will be elaborated on when optimization is discussed.

\section{Parameter Estimation}

Estimates of the kinetic parameters $E_{i}$ and $A_{i}$, and the initial mass fraction, $Y_{i, 0}$, for each component must be supplied to the optimization solver. Assuming that at each component reaction peak (denoted by subscript ${ }_{i, p}$ ), $d^{2} Y_{i} / d T^{2}=0$ and $E_{i} \gg 2 R T_{i, p}$, the estimation of the kinetic parameters 
follows from integration of Equation 3 from $Y_{i, 0}$ to $Y_{i, p}$ and $T_{0}$ to $T_{i, p}$ (Eq. 6 and 7) $[22,23]$.

$$
\begin{gathered}
E_{i} \approx \frac{e}{Y_{i, 0}} \frac{-\left.\left(\frac{d Y}{d t}\right)\right|_{T_{i, p}}}{1-Y_{f}} \frac{R T_{i, p}^{2}}{\beta} \\
A_{i} \approx \frac{e}{Y_{i, 0}} \frac{-\left.\left(\frac{d Y}{d t}\right)\right|_{T_{i, p}}}{1-Y_{f}} \frac{e^{\frac{E_{i}}{R T_{i, p}}}}{Y_{i, O_{2}}^{n_{i, O_{2}}}}
\end{gathered}
$$

The initial mass fraction for each component can be estimated as the area under the respective reaction peak, as shown in Figure 1. Trapezoidal numerical integration was used to approximate $Y_{i, 0}$.

\section{Parameter Optimization}

The system of ODEs describing the decomposition of the material is stiff and therefore is best solved with an implicit numerical method. MATLAB function 'ODE15s()' was used.

The Industrial Information and Control Centre at Auckland University of Technology developed an open source optimization interface for MATLAB, referred to as OPTI Toolbox [24]. OPTI streamlines constructing and solving linear, nonlinear, continuous and discrete optimization problems. A variety of open source and academic solvers are included. Instead of supplying analytical partial derivatives or relying on the MATLAB default method of finite-difference to determine the Jacobian, we have used a derivative free solver, NOMAD [25]. NOMAD is a mesh adaptive direct search algorithm that solves non-differentiable and global nonlinear systems.

\subsection{Constraints and Initial Conditions}

Optimization occurs over not only the kinetic parameters $E_{i}$ and $A_{i}$ but also the initial mass fractions for each component, $Y_{i, 0}$. To indicate that the 
initial condition $Y_{i, 0}$ for Equation 3 should be optimized, it was supplied to the solver as NaN. The initial conditions for Equations 4 and 5 were supplied as zero and one, respectively. Equation 5 constrains the solver to optimize the unknown initial mass fractions such that their sum is close to unity. Estimates of $E_{i}, A_{i}$, and $Y_{i, 0}$ were provided to the solver as educated guesses. Finally, a lower bound of zero was given to all optimized parameters. An upper bound of one was given to each of the optimized initial conditions.

A sensitivity analysis was performed on the optimization algorithm, the details of which can be found in the Supplementary Material. It should be noted that the algorithm performance is sensitive to the supplied initial estimations. Therefore, a proper method for estimating kinetic parameters and initial conditions is critical to a satisfactory optimized solution.

Finally, for this study, TGA mass loss data was initialized and normalized at $100{ }^{\circ} \mathrm{C}$ to ensure excess water evaporation did not impact initial weight. Justification for this starting temperature can be viewed in the Supplementary Material.

\section{Results and Discussion}

Figures 2 and 3 show the optimized simulation compared to measured normalized mass loss and the specific MLR versus temperature at a $20{ }^{\circ} \mathrm{C} / \mathrm{min}$ heating rate. Simulation results at a $10{ }^{\circ} \mathrm{C} / \mathrm{min}$ heating rate can be found in the Supplementary Material. The relative solution error $(\epsilon)$, expressed in Equation 8 as the $L^{2}$ norm of the difference between the calculated and measured solution divided by the $L^{2}$ norm of the measured solution, indicates good agreement between the simulated and measured data. The largest 
relative error is 0.0339 .

$$
\epsilon=\frac{\left\|Y_{\text {calc }}-Y_{\text {meas }}\right\|_{2}}{\left\|Y_{\text {meas }}\right\|_{2}}
$$

Moreover, the total initial mass fraction $\left(Y_{0}\right)$ remained close to unity. More specifically, the optimization algorithm kept $Y_{0}$ between 0.977 and 1.003 .

Table 1 lists the kinetic parameters and initial conditions found through optimization. Thermal decomposition of PU in an inert environment (shown in Figure 3) starts at roughly $200{ }^{\circ} \mathrm{C}$ with the pyrolysis of polyurethane into its two main constituents, isocyanate and polyol, via scission of the bond [13]. The isocyanate vaporizes after scission, while the polyol remains condensed. Above roughly $300{ }^{\circ} \mathrm{C}$ the polyol pyrolyzes. In an oxidative environment (shown in Figure 2), both the polyurethane and the melt polyol can undergo various oxidative reactions, which compete with pyrolysis since the temperature ranges associated with these reactions overlap [26]. Char is formed during the oxidative reactions, which then oxidizes around $500{ }^{\circ} \mathrm{C}$.

Comparing the two heating rates presented, the kinetic parameters reported in Table 1 are functionally equivalent, except in the case of PU in air. In an inert environment, the same pyrolysis reactions occur independent of heating rate. That is, the specific mass loss per ${ }^{\circ} \mathrm{C}$ of neat $\mathrm{PU}$ does not change with heating rate. The proposed reaction rate model also confirms that higher heating rates shift decomposition to higher temperatures due to slower reaction rates than heat transfer rates, which is observable with TGA. In contrast, extreme differences exist in an oxidative environment among various heating rates. As the heating rate decreases, the degree of oxidation of the foam and polyol melt increases, which is indicated by a more constant mass loss rate in the lower temperature range of about $200-350{ }^{\circ} \mathrm{C}$. At 
lower heating rates, the melt will primarily be volatilized at lower temperatures through oxidation instead of the pyrolysis that can occur after $300{ }^{\circ} \mathrm{C}$. There is also a higher formation of char at lower heating rates, since char is a product of these oxidative reactions. At higher heating rates, the mass loss trends approach that seen in an inert environment. The high heating rates allow pyrolysis to dominate over oxidation, which enables more of the melt to be volatilized above $300{ }^{\circ} \mathrm{C}$, but reduces the amount of thermally stable char produced. For these reasons, extracting kinetic parameters capable of describing neat PU decomposition in an oxidative environment over a large range of heating rates is not possible with any of the previously cited methods. Nevertheless, some values have been reported in literature, and have also been supplied in this study. Large variations in the mass loss trends of neat PU in an oxidative environment exist in literature. Rein et al. [13] successfully applied their kinetic parameters found at $\beta=20^{\circ} \mathrm{C} / \mathrm{min}$ to simulate 5 and $10{ }^{\circ} \mathrm{C} / \mathrm{min}$ data. However, the neat PU tested consistently underwent the same set of reactions at all three heating rates [13]. Similarly, Garrido and Font [21] successfully employed their set of kinetic parameters at $\beta=5$, 10 , and $20{ }^{\circ} \mathrm{C} / \mathrm{min}$, but their data consistently suggested a 2-step oxidative decomposition compared to 3-steps by Rein et al. [13]. In contrast, Pau [26] observed wildly different oxidative reactions occurring among $\beta=1,5,20$, and $60{ }^{\circ} \mathrm{C} / \mathrm{min}$; thereafter, he declined to find kinetic parameters due to the extreme variations observed [26]. To exemplify this point, two reactions are suggested for $\mathrm{PU}$ at $\beta=10^{\circ} \mathrm{C} / \mathrm{min}$ and three reactions are suggested for $\mathrm{PU}$ at $\beta=20^{\circ} \mathrm{C} / \mathrm{min}$ in an oxidative environment. Clearly, a single set of kinetic parameters cannot be applied to produce sufficiently accurate mass loss 
results to model smoldering over a large range of heating rates. The heating rate of the propagating front during smoldering of neat PU reportedly ranges between $1-150{ }^{\circ} \mathrm{C} / \mathrm{min}$, averaging approximately $50{ }^{\circ} \mathrm{C} / \min [13]$. Based on the average heating rate, the kinetic parameters reported at higher heating rates $\left(20^{\circ} \mathrm{C} / \mathrm{min}\right.$ or above) are better suited for modeling smoldering behavior, but still insufficient to provide any meaningful insight into the intricate chemical transitions occurring. In contrast are the kinetic parameters found

for PDA3D in air. These parameters are fairly consistent over a range of heating rates because PDA is shown to catalyze oxidation so much so that even at $\beta=20^{\circ} \mathrm{C} / \mathrm{min}, \mathrm{PDA} 3 \mathrm{D}$ has not shifted to exhibit a higher preference for pyrolysis. In other words, PDA3D is consistently undergoing the same set of reactions at different heating rates. The kinetic parameters for both neat PU and PDA3D in nitrogen are also appropriate to use over a wide range of heating rates. Again, the marginal differences in kinetic parameters reported at the two heating rates presented are functionally equivalent for modeling purposes, but parameters at higher heating rates are best for modeling combustion and flame propagation.

\subsection{Comparison of Kinetic Parameters to Those Found in Previous Studies}

Table 2 lists previously published kinetic parameters for neat PU along with the current study results for heating rates of 10 and $20{ }^{\circ} \mathrm{C} / \mathrm{min}$. Generally speaking, the first reaction listed is associated with the initial bond scission of the polyurethane and the subsequent reactions represent vaporization of the polyol melt, either by oxidation or pyrolysis. The parameters associated with char oxidation occurring above $500{ }^{\circ} \mathrm{C}$ have been omitted from the table because these values are generally not reported in literature 


\begin{tabular}{|c|c|c|c|c|c|c|c|c|c|c|c|c|c|c|}
\hline & & \multirow[b]{2}{*}{$\beta$} & \multicolumn{3}{|c|}{$1^{\text {st }} \operatorname{Rxn}$} & \multicolumn{3}{|c|}{$2^{\text {nd }} \mathrm{Rxn}$} & \multicolumn{3}{|c|}{$3^{\text {rd }} \operatorname{Rxn}$} & \multicolumn{3}{|c|}{ Char Oxidation } \\
\hline & & & $E$ & $\log _{10}(A)$ & $Y_{0}$ & $E$ & $\log _{10}(A)$ & $Y_{0}$ & $E$ & $\log _{10}(A)$ & $Y_{0}$ & $E$ & $\log _{10}(A)$ & $Y_{0}$ \\
\hline \multirow{4}{*}{ Air } & $\mathrm{PU}$ & 10 & 191 & 16.7 & 0.52 & 187 & 14.8 & 0.39 & - & - & - & 117.15 & 5.8 & 0.09 \\
\hline & & 20 & 194 & 17.2 & 0.43 & 183 & 14.5 & 0.28 & 252 & 18.8 & 0.22 & 122 & 6.0 & 0.08 \\
\hline & PDA3D & 10 & 140 & 11.4 & 0.82 & 107 & 5.4 & 0.18 & - & - & - & - & - & - \\
\hline & & 20 & 169 & 13.8 & 0.82 & 100 & 4.9 & 0.18 & - & - & - & - & - & - \\
\hline \multirow{4}{*}{$\mathrm{N}_{2}$} & $\mathrm{PU}$ & 10 & 175 & 14.4 & 0.34 & 269 & 19.4 & 0.66 & - & - & - & - & - & - \\
\hline & & 20 & 170 & 14.1 & 0.33 & 270 & 19.5 & 0.67 & - & - & - & - & - & - \\
\hline & PDA3D & 10 & 136 & 10.8 & 0.29 & 246 & 17.8 & 0.68 & - & - & - & - & - & - \\
\hline & & 20 & 144 & 11.5 & 0.29 & 247 & 17.7 & 0.69 & - & - & - & - & - & - \\
\hline
\end{tabular}

Table 1: Kinetic parameters and initial conditions determined through optimization for flexible polyurethane foam (PU) and polydopamine coated flexible polyurethane foam $(\mathrm{PDA} 3 \mathrm{D})$. Heating rate $(\beta)\left[{ }^{\circ} \mathrm{C} / \mathrm{min}\right]$, activation energy $(E)\left[\mathrm{kJ}-\mathrm{mol}^{-1}\right], \log$ of the preexponential factor $(A)\left[\log _{10}\left(\mathrm{~s}^{-1}\right)\right]$, and initial mass fraction $\left(Y_{0}\right)$, for each material component (i.e. reaction $(\operatorname{Rxn}))$.

since they have no real impact on fire development. Various graphical methods, without optimization, were used in studies [15, 18], while reaction models with optimization were used in $[12,13,11]$.

Instead of directly comparing the differences in the kinetic parameters reported, it is more useful to assess each parameter set's ability to model thermal decomposition. In fact, seemingly large differences among the reported kinetic parameters can result in very similar mass loss trends due to factors such as the kinetic compensation effect (e.g., the interdependency of $E$ and $A$ ) [13]. Using the initial conditions found through optimization and applying them to each of the proposed kinetic parameters found in literature, mass loss curves were obtained. Figures 4 and 5 show the results of solving Equation 2 using the proposed kinetic parameters from each study and the 


\begin{tabular}{cccccccccccc}
\hline & & \multicolumn{3}{c}{$\mathbf{1}^{\text {st }} \mathbf{R x n}$} & \multicolumn{3}{c}{$\mathbf{2}^{\text {nd }} \mathbf{R x n}$} & \multicolumn{3}{c}{$\mathbf{3}^{\text {rd }} \mathbf{R x n}$} \\
\hline \hline Study & $\beta$ & $E$ & $\log _{10}(A)$ & $n$ & $E$ & $\log _{10}(A)$ & $n$ & $E$ & $\log _{10}(A)$ & $n$ \\
\hline \multirow{2}{*}{ Air } & 10 & 191 & 5.0 & 13 & 72 & 3.5 & 1.3 & 63 & 2.3 & 1.2 \\
& {$[15]$} & $\mathbf{1 0}$ & $\mathbf{2 0 0}$ & $\mathbf{1 5 . 7}$ & $\mathbf{3}$ & $\mathbf{1 5 5}$ & $\mathbf{1 2 . 3}$ & $\mathbf{1}$ & $\mathbf{1 8 5}$ & $\mathbf{1 3 . 6}$ & $\mathbf{1}$ \\
& Roberts et al. & $\mathbf{1 0}$ & $\mathbf{1 9 1}$ & $\mathbf{1 6 . 7}$ & $\mathbf{1}$ & $\mathbf{1 8 7}$ & $\mathbf{1 4 . 8}$ & $\mathbf{1}$ & - & - & - \\
& {$[15]$} & 20 & 163 & 21.2 & 19 & 102 & 2.9 & 2.2 & 80 & 2.2 & 1.3 \\
& {$[\mathbf{1 3}]$} & $\mathbf{2 0}$ & $\mathbf{1 4 8}$ & $\mathbf{1 1 . 3}$ & $\mathbf{0 . 2 1}$ & $\mathbf{1 9 4}$ & $\mathbf{1 5 . 4}$ & $\mathbf{0 . 5 2}$ & $\mathbf{2 0 1}$ & $\mathbf{1 5 . 2}$ & $\mathbf{1 . 2 3}$ \\
& Roberts et al. & $\mathbf{2 0}$ & $\mathbf{1 9 4}$ & $\mathbf{1 7 . 2}$ & $\mathbf{1}$ & $\mathbf{1 8 3}$ & $\mathbf{1 4 . 5}$ & $\mathbf{1}$ & $\mathbf{2 5 2}$ & $\mathbf{1 8 . 8}$ & $\mathbf{1}$ \\
\hline & {$[\mathbf{1 1}]$} & $\mathbf{( 5 , 1 0 )}$ & $\mathbf{1 3 5}$ & $\mathbf{8 . 2}$ & $\mathbf{1}$ & $\mathbf{1 7 5}$ & $\mathbf{9 . 9}$ & $\mathbf{1 . 1 6}$ & - & - & - \\
& Roberts et al. & $\mathbf{1 0}$ & $\mathbf{1 7 5}$ & $\mathbf{1 4 . 4}$ & $\mathbf{1}$ & $\mathbf{2 6 9}$ & $\mathbf{1 9 . 4}$ & $\mathbf{1}$ & - & - & - \\
& {$[15]$} & 10 & 112 & 17.3 & 15.4 & 62 & 2.8 & 1 & - & - & - \\
$\mathrm{N}_{2}$ & 20 & 120 & 22.4 & 18.4 & 54 & 2.3 & 0.9 & - & - & - \\
& {$[15]$} & 20 & 178 & 14.3 & 9.51 & 262 & 19.1 & 1.23 & - & - & - \\
& {$[18]$} & 20 & 121 & 8.9 & 6.56 & 253 & 18.4 & 1.19 & - & - & - \\
& {$[18]$} & $\mathbf{2 0}$ & $\mathbf{1 4 8}$ & $\mathbf{1 1 . 3}$ & $\mathbf{0 . 2 1}$ & $\mathbf{1 2 4}$ & $\mathbf{8 . 2}$ & $\mathbf{1 . 1 4}$ & - & - & - \\
& {$[13]$} & $\mathbf{2 0}$ & $\mathbf{1 7 0}$ & $\mathbf{1 4 . 1}$ & $\mathbf{1}$ & $\mathbf{2 7 0}$ & $\mathbf{1 9 . 5}$ & $\mathbf{1}$ & - & - & - \\
\hline
\end{tabular}

Table 2: Comparison of published kinetic parameters of decomposition in air and $\mathrm{N}_{2}$ of neat PU. Heating rate $(\beta)\left[{ }^{\circ} \mathrm{C} / \mathrm{min}\right]$, activation energy $(E)\left[\mathrm{kJ}-\mathrm{mol}^{-1}\right]$, log of the preexponential factor $(A)\left[\log _{10}\left(\mathrm{~s}^{-1}\right)\right]$, and reaction order $(n)$. Reaction $(\mathrm{Rxn})$. Parenthesis denotes use of multiple heating rates in calculation. Optimized models indicated in bold.

herein optimized initial conditions. It should be noted that, in an oxidative environment, $\mathrm{O}_{2}$ concentrations were only applied in Equation 2 to studies that considered it in their formulation of kinetic parameters (this study and $[12,13])$.

Good agreement exists between the PU results of this study and the PU results obtained by optimization in $[12,13]$, however, the result from opti- 
mization in [11] does not produce a comparable mass loss curve. The graphical methods generally do not reproduce the TGA data well. This analysis elucidates the need to confirm that reported kinetic parameters can functionally describe the mass loss observed in TGA. Undoubtedly, there should be slight variations in simulated results (and kinetic parameter sets) due to slight differences in samples and experimental setup, but ultimately the mass loss curves should be comparable. That is not the case for the majority of graphical methods presented.

\subsection{Comparison Between Halophosphate and Polydopamine Flame Retar- dant Polyurethane}

Previous studies analyzed PU containing a halophosphate FR additive $[15,18,21]$. Two flame retarding mechanisms are accomplished with this additive. As a halophosphate decomposes, it produces either chlorine ([18, 21]) or bromine ([15]) vapors. These vapors will neutralize free radicals formed as the plastic decomposes, which reduces the volatiles available for combustion [18]. The second mechanism acts in the condensed-phase as phosphoric acid is formed. The acid catalyzes the decomposition reaction but also dehydrates the plastic, which subsequently causes the formation of an insulating char $[18,26]$. An additional FR, melamine, was present in the PU studied by Pau et al. [18], Pau [26]. Melamine acts in both the gas and condensed-phase. As melamine degrades, it releases non-combustible gases, e.g. ammonia and nitrogen, which dilute combustible concentrations. The production of ammonia catalyzes the decomposition of $\mathrm{PU}$, but melamine also reacts with

the phosphoric acid and isocyanate, forming different types of cross-linked residues at specific temperatures $[18,26]$. In summary, halophosphate FR 
PU will begin decomposing at a lower temperature compared to neat PU, which allows FR mechanisms to act in the gas-phase, but the subsequent activation of the FR in the condensed-phase will form a protective residue char that slows further decomposition. Melamine complements these halophosphate FR mechanisms.

Figure 6 contains data from Pau [26], Pau et al. [18] and shows a comparison between neat PU, halophosphate-melamine FR PU (HPMPU), and PDA3D in both air and nitrogen at $\beta=20{ }^{\circ} \mathrm{C} / \mathrm{min}$. Although the neat PU used by Pau [26] is nominally comparable to that used in this study, there are notable differences in the TGA behavior, especially in a nitrogen environment. This could be due to small but conflating factors, such as the specific chemical structure of the foam, density, sample size, experimental setup, and equipment calibration. Although in Pau [26] and in this study the results are repeatable, the significant differences in the neat foams do not allow for direct comparison between the halophosphate-melamine and PDA treated foams. Therefore, the following discussion will only note the variation between the FR PU and the neat PU from the respective study. Qualitative conclusions will then be drawn regarding the effectiveness and mechanisms of PDA as compared to halophosphate-melamine. First, the differences observed by Pau [26] between HPMPU and PU in nitrogen will be examined, followed by those observed between PDA3D and PU. Next, the dissimilarities in air between HPMPU and PU will be considered, followed by analysis of those observed between PDA3D and PU. Table 3 summarizes the differences in TGA data subsequently discussed, which is limited to observations at $\beta=20{ }^{\circ} \mathrm{C} / \mathrm{min}$. The reader is again cautioned that direct quantitative 
comparisons between HPMPU and PDA3D should not be made due to the discrepancy between the neat foam samples used in this study and Pau [26]. The bounding temperatures of a reaction region are defined as the points at which the specific mass loss per ${ }^{\circ} \mathrm{C}$ equals 0.002 .

The onset of the first reaction for HPMPU begins about $7{ }^{\circ} \mathrm{C}$ below that for neat PU in nitrogen. The initial mass loss rate and temperature span of the first reaction is larger for HPMPU. Furthermore, the peak mass loss rate of HPMPU occurs $13{ }^{\circ} \mathrm{C}$ below that of PU, but the magnitudes are comparable. Finally, the total mass lost during the first reaction is $17 \%$ higher for HPMPU than PU. The onset of the second reaction in HPMPU begins about $11{ }^{\circ} \mathrm{C}$ above that in neat PU. However, unlike in the first reaction, the mass loss rate and temperature span is comparable between the FR and neat foam. The peak mass loss rate for HPMPU occurs $5{ }^{\circ} \mathrm{C}$ above that for PU, and the magnitude is slightly lower. Finally, the total mass lost during the second reaction is $15 \%$ higher for PU than HPMPU. These findings are consistent with the flame retardant mechanisms of halophosphate and melamine. That is, they catalyze the first decomposition reaction of the foam in order to act in the gas-phase, while also changing the condensed phase chemistry, forming residue which prevents later decomposition during the second reaction.

The onset of the first reaction for PDA3D begins about $9{ }^{\circ} \mathrm{C}$ above that for neat PU in nitrogen. The initial mass loss rate is larger for the neat foam but the temperature span is comparable to PDA3D. Moreover, the peak mass loss rate for PDA3D occurs $5{ }^{\circ} \mathrm{C}$ above that for $\mathrm{PU}$ and the magnitude is notably smaller. Finally, the total mass lost during the first reaction is $25 \%$ higher for PU than PDA3D. The onset of the second reaction in PDA3D be- 
gins about $4{ }^{\circ} \mathrm{C}$ below that in $\mathrm{PU}$. However, unlike in the first reaction, both the mass loss rate and temperature span are comparable between the FR and neat foam. The peak mass loss rate occurs at nearly the same temperature for PU and PDA3D, but the magnitude is slightly higher for the neat foam. Finally, the total mass lost during the second reaction is $9 \%$ higher for $\mathrm{PU}$ than PDA3D.

The description of the first reaction of PDA3D is in direct contrast to that of the halophosphate-melamine treated foam. That is, the PDA delays the initial reaction and it diminishes the total mass lost. Furthermore, although the PDA appears to catalyze the second reaction, it otherwise appears to impact the second reaction in a similar manner to a halophosphate-melamine FR. One hypothesis is that the PDA predominately acts in the condensedphase by first creating a thermal barrier, protecting PU from decomposition. Unlike the halophosphate-melamine FR, this protective layer resists initial decomposition and could possibly include thermal stability from chemical bonding during deposition. Once the initial decomposition of the foam occurs, the PDA then acts similarly to a halophosphate-melamine FR in that it, again acting primarily in the condensed-phase, forms stable cross-linked residues which slow decomposition.

The onset of the main decomposition region in air, where competing pyrolysis and oxidative reactions occur, begins at roughly the same temperature for HPMPU and neat PU, but the temperature span is $36{ }^{\circ} \mathrm{C}$ larger for $\mathrm{PU}$. Additionally, the peak mass loss rate for HPMPU occurs $6{ }^{\circ} \mathrm{C}$ below that for PU, but the magnitudes are comparable. Finally, the total mass lost during the main decomposition region is $16 \%$ higher for PU than HPMPU. The 
earlier decomposition of HPMPU seen in nitrogen is no longer present in an oxidative environment since oxidative reactions with $\mathrm{PU}$ occur over the same temperature range [26]. However, the gas-phase action of the FR is likely still impactful. The halophosphate-melamine also still increases thermally stable char production, reducing the decomposition region temperature span and mass loss.

The onset of the main decomposition region in air for PDA3D begins 19 ${ }^{\circ} \mathrm{C}$ above that of neat $\mathrm{PU}$ and the temperature span is $68^{\circ} \mathrm{C}$ larger for $\mathrm{PU}$. Furthermore, the peak mass loss rate for PDA3D occurs at a temperature $32{ }^{\circ} \mathrm{C}$ above that for PU, but the magnitude is also larger. Finally, the total mass lost during the main decomposition region is $18 \%$ higher for $\mathrm{PU}$ than PDA3D.

The description of the main decomposition region of PDA3D has notable differences to that of the halophosphate-melamine treated foam. PDA3D significantly delays decomposition and more effectively limits mass loss via higher char production than HPMPU. Again, it appears as though PDA acts more predominately in the condensed-phase than its halophosphatemelamine counterpart. Although PDA is susceptible to oxidation, it still delays the release of vaporized combustibles and contributes to higher char formation, both indicative of its effectiveness as a flame retardant. PDA also appears to be more effective at these particular FR mechanisms than the commercially available halophosphate-melamine example given. However, it should be noted that the mass loss trends in air for PDA3D at $\beta=20{ }^{\circ} \mathrm{C} / \mathrm{min}$ are similar to those observed by Pau [26] for HPMPU at $\beta=5{ }^{\circ} \mathrm{C} / \mathrm{min}$. What cannot be fully determined from examining the TGA data is the impact that 
PDA has on gas-phase reactions, although it can be concluded that it does not volatilize in the same manner as a halophosphate.

\begin{tabular}{|c|c|c|c|c|c|c|}
\hline \multirow{2}{*}{$\begin{array}{c}\text { Environment } \\
\text { Air }\end{array}$} & \multicolumn{6}{|c|}{ Decomposition Region - Multiple Rxns } \\
\hline & \multicolumn{2}{|c|}{$T$ Range $\left[{ }^{\circ} \mathrm{C}\right]$} & \multicolumn{2}{|r|}{$T_{p}$} & \multicolumn{2}{|c|}{ Mass Lost } \\
\hline PDA3D & \multicolumn{2}{|c|}{$264-357(\Delta 93)$} & \multicolumn{2}{|r|}{315} & \multicolumn{2}{|r|}{$73 \%$} \\
\hline HPMPU* & \multicolumn{2}{|c|}{$252-369(\Delta 117)$} & \multicolumn{2}{|r|}{275} & \multicolumn{2}{|r|}{$76 \%$} \\
\hline $\mathrm{PU}$ & \multicolumn{2}{|c|}{$245-406(\Delta 161)$} & \multicolumn{2}{|r|}{283} & \multicolumn{2}{|r|}{$86 \%$} \\
\hline $\mathrm{PU}^{*}$ & \multicolumn{2}{|c|}{$250-403(\Delta 153)$} & \multicolumn{2}{|r|}{281} & \multicolumn{2}{|r|}{$88 \%$} \\
\hline Environment & \multicolumn{3}{|c|}{ 1st Rxn } & \multicolumn{3}{|c|}{ 2nd Rxn } \\
\hline $\mathbf{N}_{2}$ & $T$ Range $\left[{ }^{\circ} \mathrm{C}\right]$ & $T_{p}$ & Mass Lost & $T$ Range $\left[{ }^{\circ} \mathrm{C}\right]$ & $T_{p}$ & Mass Lost \\
\hline PDA3D & $263-314(\Delta 51)$ & 299 & $20 \%$ & $353-420(\Delta 67)$ & 390 & $56 \%$ \\
\hline HPMPU* & $251-306(\Delta 55)$ & 281 & $21 \%$ & $351-424(\Delta 73)$ & 391 & $62 \%$ \\
\hline $\mathrm{PU}$ & $254-307(\Delta 53)$ & 294 & $25 \%$ & $357-424(\Delta 67)$ & 391 & $61 \%$ \\
\hline $\mathrm{PU}^{*}$ & $258-304(\Delta 46)$ & 294 & $18 \%$ & $340-415(\Delta 75)$ & 386 & $71 \%$ \\
\hline
\end{tabular}

Table 3: Comparison of neat PU, halophosphate-melamine flame retardant flexible polyurethane foam (HPMPU), and polydopamine coated flexible polyurethane foam (PDA3D) in air and $\mathrm{N}_{2}, \beta=20{ }^{\circ} \mathrm{C} / \mathrm{min} . T_{p}$ denotes temperatures at the maximum mass loss rate. ${ }^{*} \mathrm{Pau}[26]$, Pau et al. [18].

\subsection{Hypothetical-Difference Analysis of Polydopamine Flame Retardant Polyurethane}

As previously stated, the PDA nanocoating is $15.9 \mathrm{wt} \%$ of the coated PU. Therefore, to understand how PDA and PU interact as they thermally degrade together, a hypothetical composite of the material was constructed from separate TGA runs of neat PDA and neat PU. From that, the data were combined to obtain a hypothetical mass loss curve, which represents PDA and PU degrading together without any interaction (PDA+PU, Eq. 9). This 
hypothetical mass loss was then subtracted from the mass loss data of PDA coated PU (PDA3D) to quantify the differences between the two (Eq. 10). Figure 7 illustrates the difference between PDA3D and combined TGA data for PDA and PU at $\beta=20{ }^{\circ} \mathrm{C} / \mathrm{min}$. TGA data for neat PDA can be found in the Supplementary Material.

$$
\begin{gathered}
(Y)_{P D A+P U}=(Y)_{P D A} \times 0.159+(Y)_{P U} \times(1-0.159) \\
\Delta Y=(Y)_{P D A 3 D}-(Y)_{P D A+P U}
\end{gathered}
$$

In nitrogen at a heating rate of $20^{\circ} \mathrm{C} / \mathrm{min}$, the PDA3D initially retains more mass than PDA+PU until $378^{\circ} \mathrm{C}$ with a maximum of about $6 \%$ more between $285-365{ }^{\circ} \mathrm{C}$. From this point until roughly $430{ }^{\circ} \mathrm{C}$, PDA3D retains less mass than $\mathrm{PDA}+\mathrm{PU}$, with a maximum of $13 \%$ less at $406{ }^{\circ} \mathrm{C}$. After this point, both specimens continue to lose mass at roughly the same rate through the remainder of the experiment. Considering these results with the analysis presented in the previous section, PDA consistently reduces the amount of combustible products released from PU up to about midway through the second reaction, in spite of catalyzing the second reaction. This is a considerable benefit from a flammability perspective.

The differences in air are much more pronounced. The PDA3D loses mass more slowly than the PDA+PU hypothetical composite until $292^{\circ} \mathrm{C}$. At that point, PDA3D has $23 \%$ more mass than PDA+PU. After this point, PDA3D starts to rapidly lose mass at a higher rate than $\mathrm{PDA}+\mathrm{PU}$ and at $314^{\circ} \mathrm{C}$ reaches the same mass as PDA+PU. PDA3D continues at a higher MLR until $338^{\circ} \mathrm{C}$, at which point the PDA3D has $37 \%$ less mass than the PDA+PU. Then, the PDA+PU returns to a higher MLR than PDA3D until about 420 
${ }^{\circ} \mathrm{C}$, after which point both specimens continue to lose mass at roughly the same rate until neither has any mass remaining. Considering these results with the analysis presented in the previous section, PDA significantly reduces the amount of combustible products released from PU up to about midway through the reaction region where PU bond scissions are occurring. This is beneficial from a flammability perspective in that PDA can greatly diminish combustible gases during the early stages of heating. However, PDA does appear to catalyze subsequent oxidation in the temperature region before the polyol melt would normally be consumed via pyrolysis. This "premature" release of gaseous products surely cannot be beneficial from a flammability perspective, however, gas analysis (which is not presented in this work) would elucidate whether PDA is simultaneously acting as a gas-phase combustion inhibitor.

The differences between the PDA3D and PDA+PU hypothetical curves suggest that the mechanism behind PDA as a flame retardant is not a simple additive effect. While some of the retardancy is likely from protective char formation, it is also conceivable that a chemical reaction is aiding the performance. One hypothesis is that as isocyanate decomposes and volatilizes, diffusion of this isocyanate into the polydopamine coating leads to further cross-linking and additional protection during the initial stages of PU decomposition. However, as the temperature continues to increase, the protective coating eventually deteriorates and can no longer contain the degradation products, leading to comparatively large second stage mass loss, which occurs at a significantly lower temperature in an oxidative environment. Chemical analysis comparing the stages of degradation of neat PU to polydopamine 
coated foam should be performed to verify the condensed-phase mechanisms of PDA flame retardancy.

\section{Conclusion}

A novel flame retardant nanocoating of polydopamine was applied to flexible polyurethane foam and thermogravimetrically analyzed. Thermal degradation kinetics were described by a simplified multi-component, Arrhenius expression coupled with a first-order reaction model. A mesh adaptive direct search algorithm was used to optimize for kinetic parameters and initial mass fractions, which was better able to simulate the TGA data compared to graphical methods and easier to implement than more complex optimized models. However, none of the cited models consistently describe PU decomposition in air over a large range of heating rates due to the complexity of competing oxidative and pyrolysis reactions. In contrast, the kinetic parameters found for PDA3D in air are fairly consistent over a range of heating rates because PDA is shown herein to catalyze oxidation so much so that even at $\beta=20^{\circ} \mathrm{C} / \mathrm{min}$, PDA3D has not shifted to exhibit a higher preference for pyrolysis. The kinetic parameters for both neat PU and PDA3D in nitrogen are also appropriate to use over a wide range of heating rates. The marginal differences reported at the two heating rates presented are functionally equivalent when applied, but parameters at higher heating rates are best for modeling flame propagation.

A hypothetical composite of the PDA coated PU was constructed from separate TGA runs of neat PDA and neat PU. These data were then subtracted from the actual polydopamine coated flexible polyurethane foam 
(PDA3D) TGA data to visualize how PDA affects PU as it thermally degrades. In nitrogen, PDA delays the first reaction that breaks the polyurethane bonds and it diminishes the total mass lost, which cannot be said for a halophosphate flame retardant. Additionally, although the PDA catalyzes the second reaction, it ultimately mimics a halophosphate-melamine FR by forming stable cross-linked residues that slow and reduce decomposition during the second reaction. In summary, PDA consistently reduces the amount of combustible products released from PU up to about midway through the second reaction. This is a considerable benefit from a flammability perspective. In air, PDA significantly delays decomposition and more effectively limits mass loss via higher char production than the commercially available halophosphate-melamine treated foam discussed. In fact, PDA significantly reduces the amount of combustible products released from PU up to about midway through the reaction region where PU bond scissions are occurring. This is beneficial from a flammability perspective in that PDA can greatly diminish combustible gases during the early stages of heating. However, PDA does appear to catalyze subsequent oxidation in the temperature region before the polyol melt would normally be consumed via pyrolysis, which indicates a "premature" release of gaseous, and likely combustible, products. The hypothetical-difference analysis suggests that the mechanism behind PDA as a flame retardant is not a simple additive effect. One hypothesis is that as isocyanate decomposes and volatilizes, diffusion of this isocyanate into the polydopamine coating leads to further cross-linking and additional protection during the initial stages of PU decomposition. However, as the temperature continues to increase, the protective coating eventually deteriorates and can 
no longer contain the degradation products, leading to comparatively large second stage mass loss, which occurs at a significantly lower temperature in an oxidative environment.

\section{Acknowledgments}

The work presented in this paper was supported in part by the National Science Foundation (NSF) through the Integrative Graduate Education and Research Traineeship (IGERT) program. We also gratefully acknowledge the Robert A. Welch Foundation (Grant no. F-1709), DuPont Young Professor Award, the Norman Hackerman Advanced Research Program (Grant no. ARP-003658-0037-2011), and the National Science Foundation CAREER Award (Grant no. DMR-1053293) for partial financial support. 


\section{Glossary}

\section{Acronyms}

DOPA dihydroxyphenylalanine

EPA U.S. Environmental Protection Agency

FDS Fire Dynamics Simulator

FR flame retardant

GA genetic algorithm

HPMPU halophosphate-melamine flame retardant flexible polyurethane foam

MCC microscale combustion calorimetry

MLR mass loss rate

PDA polydopamine

PDA3D polydopamine coated flexible polyurethane foam

PFA Polyurethane Foam Association

PU flexible polyurethane foam

$\mathbf{R x n}$ reaction

TDI toluene diisocyanate

TGA thermogravimetric analysis

\section{Symbols}


$A$ pre-exponential factor

$\alpha$ mass fraction decomposed

$E$ activation energy

$\epsilon$ relative solution error

$\beta$ heating rate

$m$ instantaneous mass

$n$ reaction order

$\mathbf{N}_{2}$ nitrogen

$\mathrm{O}_{2}$ oxygen

$R$ universal gas constant

$f(\alpha)$ reaction model

$T$ temperature

$t$ time

$Y$ mass fraction

\section{Subscripts}

0 initial

calc calculated value

$f$ final 
$i$ reaction index

meas measured value

$\mathrm{O}_{2}$ atmospheric oxygen

$p$ reaction peak

$r$ residual

\section{References}

[1] C. J. Hilado, Flammability handbook for plastics, CRC Press, 1998.

[2] P. F. Association, et al., Flexible polyurethane foam: Industry at a glance, 2007.

[3] EPA 744-R-15-002, Flame Retardants Used in Flexible Polyurethane Foam: An Alternatives Assessment Update, Technical Report, U.S. Environmental Protection Agency, 2015.

[4] D. R. Dreyer, D. J. Miller, B. D. Freeman, D. R. Paul, C. W. Bielawski, Perspectives on poly(dopamine), Chem. Sci. 4 (2013) 3796-3802.

[5] M. Zammarano, D. Fox, P. Grützmacher, R. Davis, Flame retardant coatings, 2015. URL: https://www.google.com/patents/ US20150073071, uS Patent App. 14/336,581.

[6] J. H. Cho, V. Vasagar, K. Shanmuganathan, A. R. Jones, S. Nazarenko, C. J. Ellison, Bioinspired Catecholic Flame Retardant Nanocoating for Flexible Polyurethane Foams, Chem. Mater. 27 (2015) 6784-6790. 
[7] A. Khawam, D. R. Flanagan, Solid-state kinetic models: basics and mathematical fundamentals, The journal of physical chemistry B 110 (2006) 17315-17328.

[8] L. Shi, M. Y. L. Chew, A review of fire processes modeling of combustible materials under external heat flux, Fuel 106 (2013) 30-50.

[9] I. T. Leventon, J. Li, S. I. Stoliarov, A flame spread simulation based on a comprehensive solid pyrolysis model coupled with a detailed empirical flame structure representation, Combust. Flame 162 (2015) 3884-3895.

[10] T. H. Tsai, M. J. Li, I. Y. Shih, R. Jih, S. C. Wong, Experimental and numerical study of autoignition and pilot ignition of PMMA plates in a cone calorimeter, Combust. Flame 124 (2001) 466-480.

[11] K. Prasad, R. Kramer, N. Marsh, M. Nyden, Numerical simulation of fire spread on polyurethane foam slabs, Fire Mater. (2009) 697 - 708.

[12] G. Rein, A. Bar-Ilan, a. C. Fernandez-Pello, J. L. Ellzey, J. L. Torero, D. L. Urban, Modeling of one-dimensional smoldering of Polyurethane in microgravity conditions, Proc. Combust. Inst. 30 II (2005) 2327-2334.

[13] G. Rein, C. Lautenberger, A. C. Fernandez-Pello, J. L. Torero, D. L. Urban, Application of genetic algorithms and thermogravimetry to determine the kinetics of polyurethane foam in smoldering combustion, Combust. Flame 146 (2006) 95-108.

[14] F. Rogers, T. Ohlemiller, Pyrolysis kinetics of a polyurethane foam by thermogravimetry; a general kinetic method, Journal of Macromolecular ScienceChemistry 15 (1981) 169-185. 
[15] C. Y. H. Chao, J. H. Wang, Comparison of the Thermal Decomposition Behavior of a Non-Fire Retarded and a Fire Retarded Flexible Polyurethane Foam with Phosphorus and Brominated Additives, J. Fire Sci. 19 (2001) 137-156.

[16] H. L. Friedman, Kinetics of thermal degradation of char-forming plastics from thermogravimetry. Application to a phenolic plastic, J. Polym. Sci. Part C Polym. Symp. 6 (1964) 183-195.

[17] R. Bilbao, J. F. Mastral, J. Ceamanos, M. E. Aldea, Kinetics of the thermal decomposition of polyurethane foams in nitrogen and air atmospheres, J. Anal. Appl. Pyrolysis 37 (1996) 69-82.

[18] D. S. W. Pau, C. M. Fleischmann, M. J. Spearpoint, K. Y. Li, Determination of kinetic properties of polyurethane foam decomposition for pyrolysis modelling, J. Fire Sci. 31 (2013) 356-384.

[19] K. B. McGrattan, S. Hostikka, J. E. Floyd, Fire dynamics simulator, users guide, NIST special publication 1019 (2010).

[20] C. Lautenberger, A generalized pyrolysis model for combustible solids, Dissertation, University of California, Berkeley, 2007.

[21] M. A. Garrido, R. Font, Pyrolysis and combustion study of flexible polyurethane foam, J. Anal. Appl. Pyrolysis 113 (2015) 202-215.

[22] R. E. Lyon, N. Safronava, E. Oztekin, A Simple Method for Determining Kinetic Parameters for Materials in Fire Models, Fire Saf. Sci. 10 (2011) $765-777$. 
[23] K. McGrattan, S. Hostikka, Randall McDermott, J. Floyd, C. Weinschenk, K. Overholt, Fire Dynamics Simulator Technical Reference Guide Volume 2: Verification, Technical Report, NIST, 2015. doi:10. 6028/NIST .SP.1018-2.

[24] J. Currie, D. I. Wilson, OPTI: Lowering the Barrier Between Open Source Optimizers and the Industrial MATLAB User, in: N. Sahinidis, J. Pinto (Eds.), Foundations of Computer-Aided Process Operations, Savannah, Georgia, USA, 2012.

[25] M. Abramson, C. Audet, G. Couture, J. Dennis, Jr., S. Le Digabel, C. Tribes, The NOMAD project, Software available at https://www. gerad.ca/nomad/, 2016.

[26] D. S. W. Pau, A comparative study on combustion behaviours of polyurethane foams with numerical simulations using pyrolysis models, Dissertation, University of Canterbury. Civil and Natural Resources Engineering, 2013. 


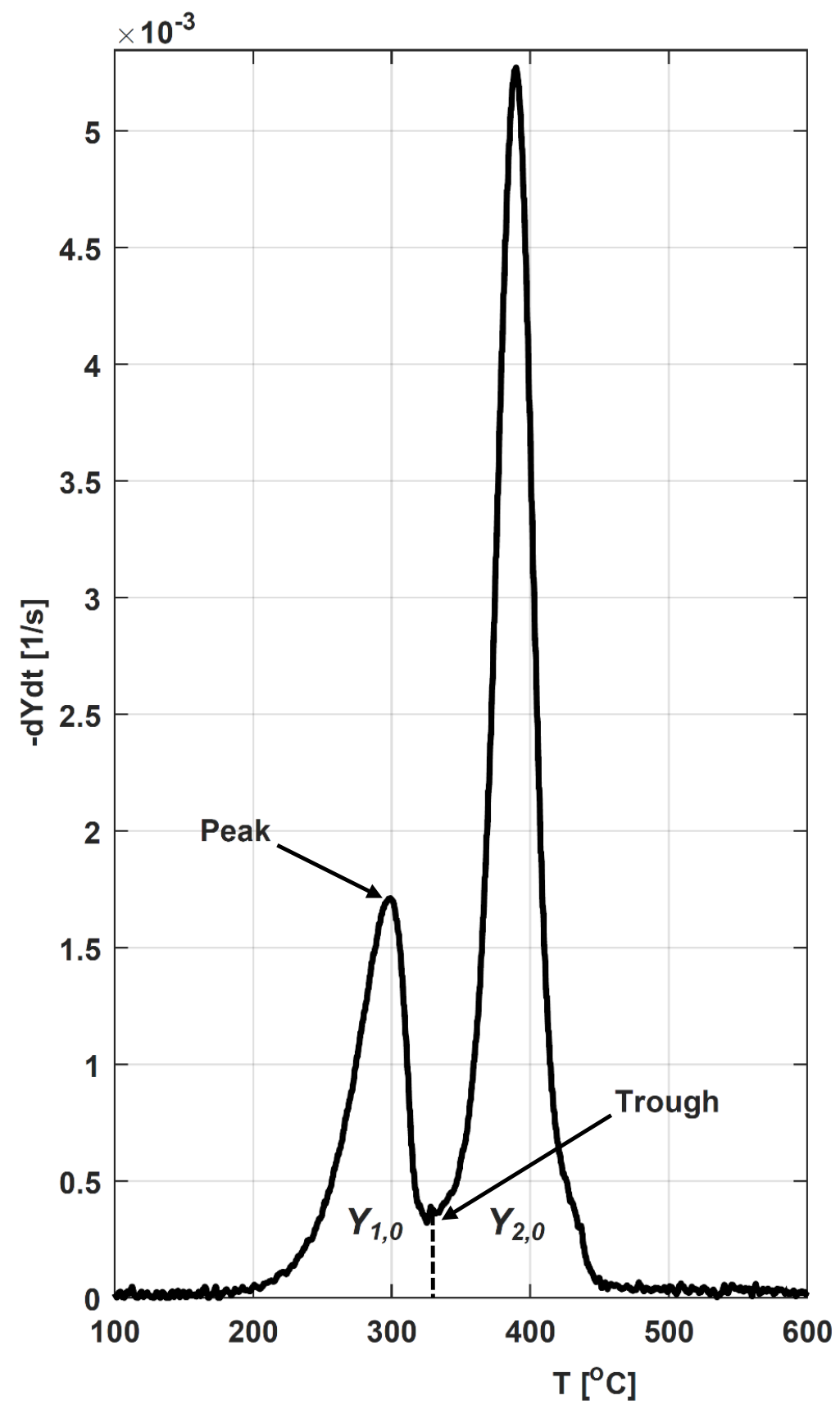

Figure 1: Representative specific MLR curve showing two separate reactions. Initial mass fractions for each material component is estimated by calculating the area under its respective reaction peak. 

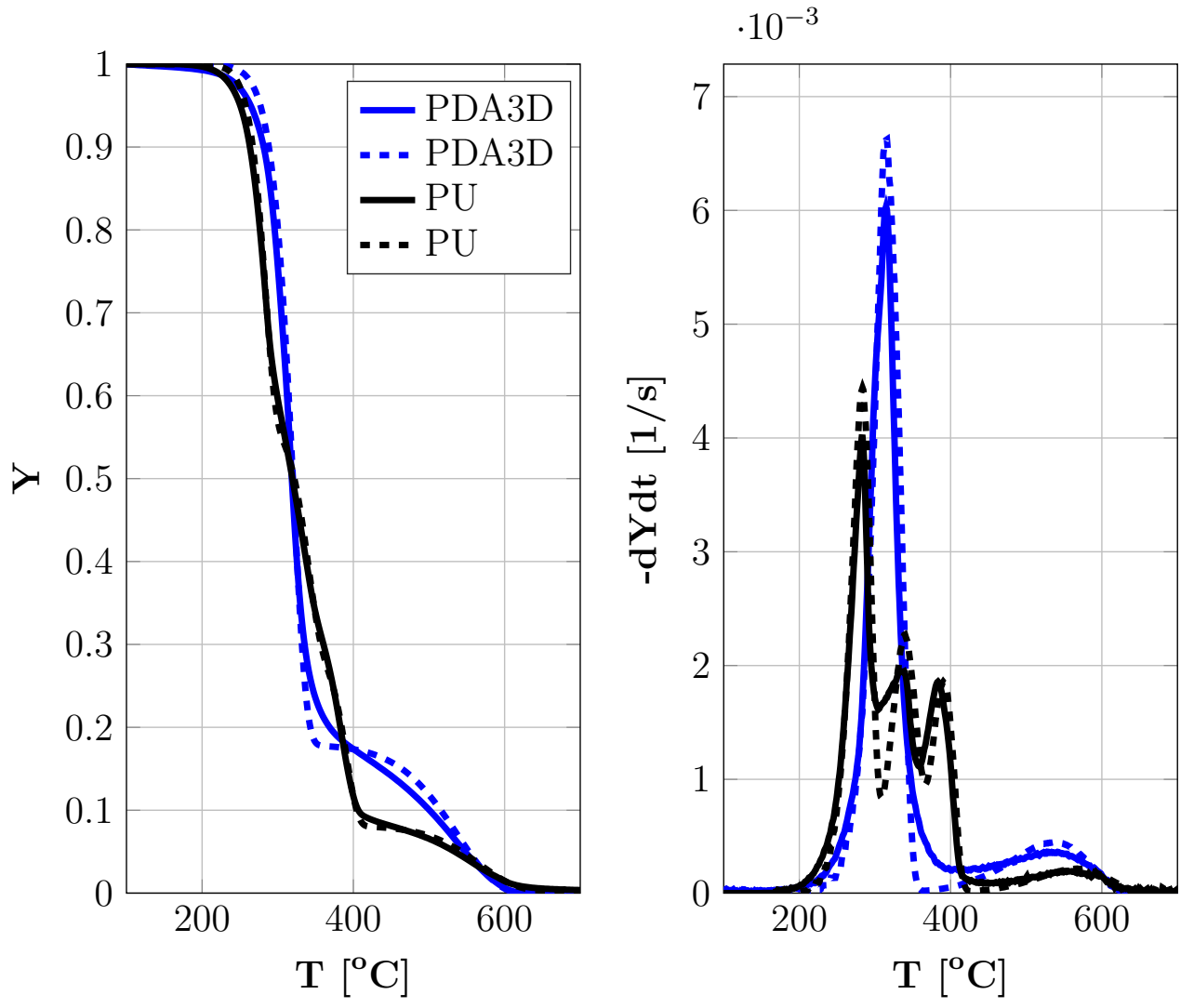

Figure 2: Measured and simulated mass fraction and specific MLR vs. temperature. Solid lines: measured; dashed lines: simulation. Air, $\beta=20^{\circ} \mathrm{C} / \mathrm{min}$. 

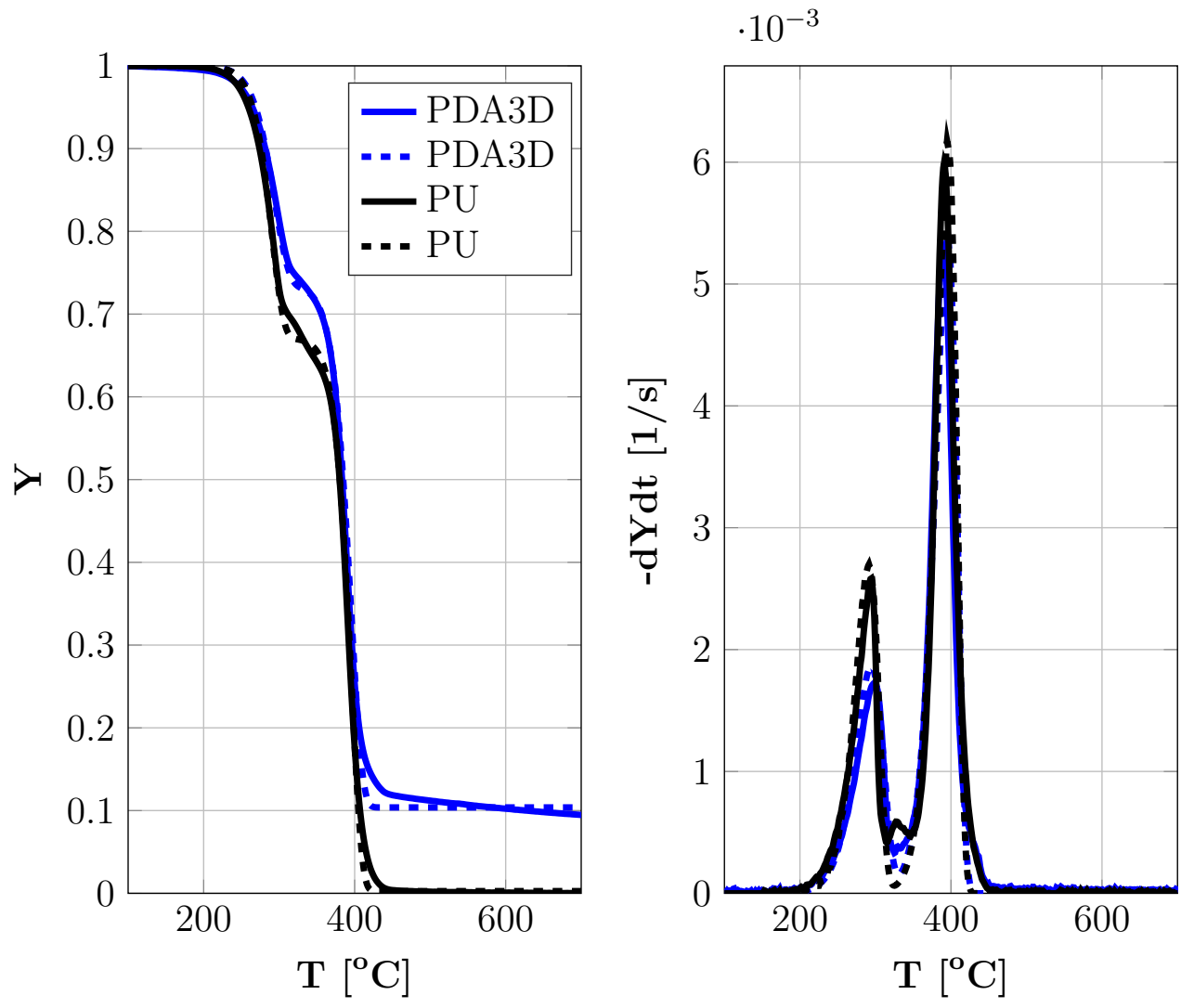

Figure 3: Measured and simulated mass fraction and specific MLR vs. temperature. Solid lines: measured; dashed lines: simulation. $\mathrm{N}_{2}, \beta=20{ }^{\circ} \mathrm{C} / \mathrm{min}$. 


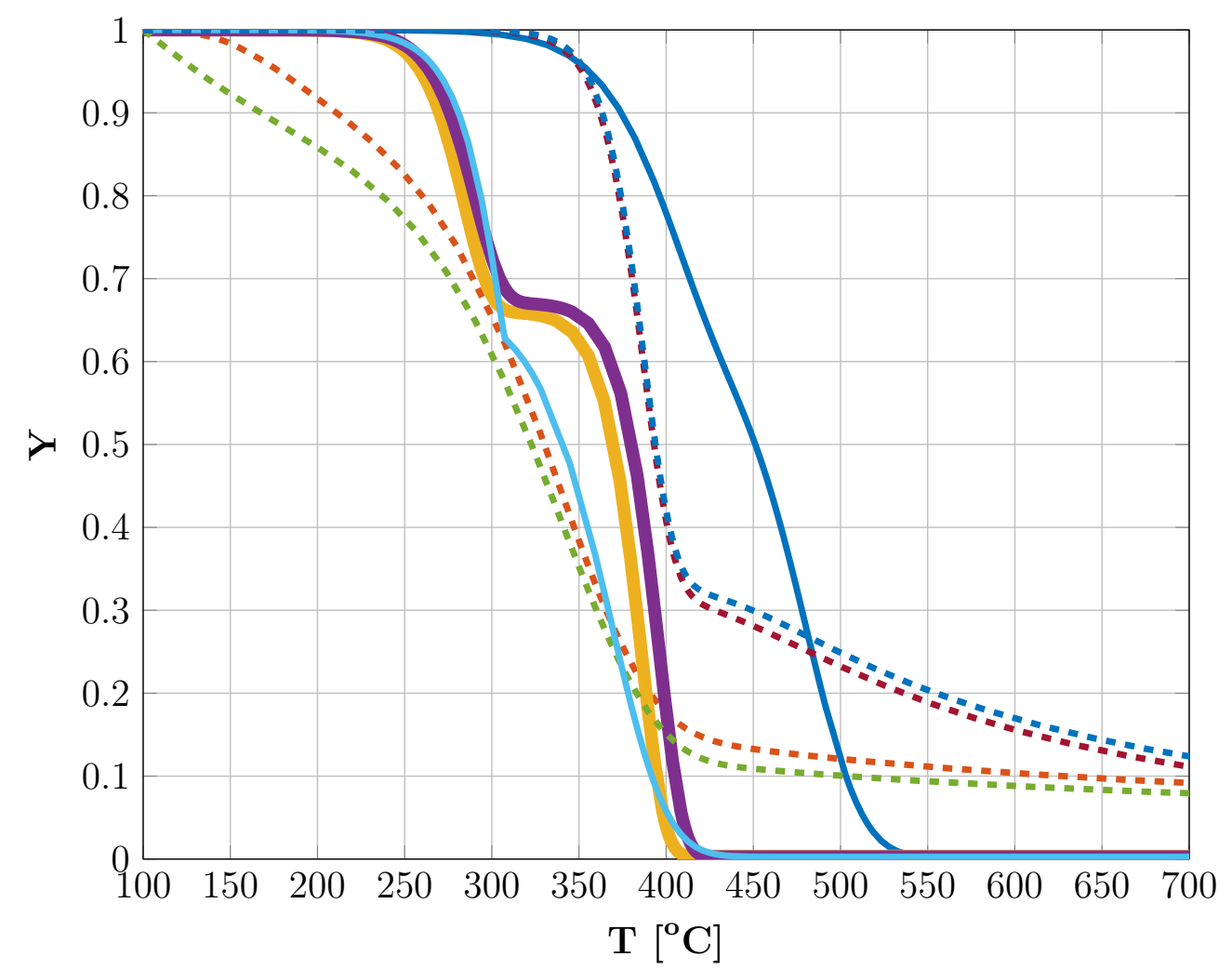

— Prasad et al. $2009 ; \beta=10$

- - Chao \& Wang 2001; $\beta=10$

Roberts et al.; $\beta=10$

Roberts et al.; $\beta=20$

- - Chao \& Wang 2001; $\beta=20$

- Rein et al. $2006 ; \beta=20$

- - Pau et al. 2013; $\beta=20$

-.- Pau et al. 2013; $\beta=20$

Figure 4: Comparison of simulated TGA results using kinetic parameters from literature. PU, $\mathrm{N}_{2}$. Solid lines: kinetic parameters from optimized models; dashed lines: kinetic parameters from graphical methods. This study is indicated with bold lines. $\beta\left[{ }^{\circ} \mathrm{C} / \mathrm{min}\right]$. 


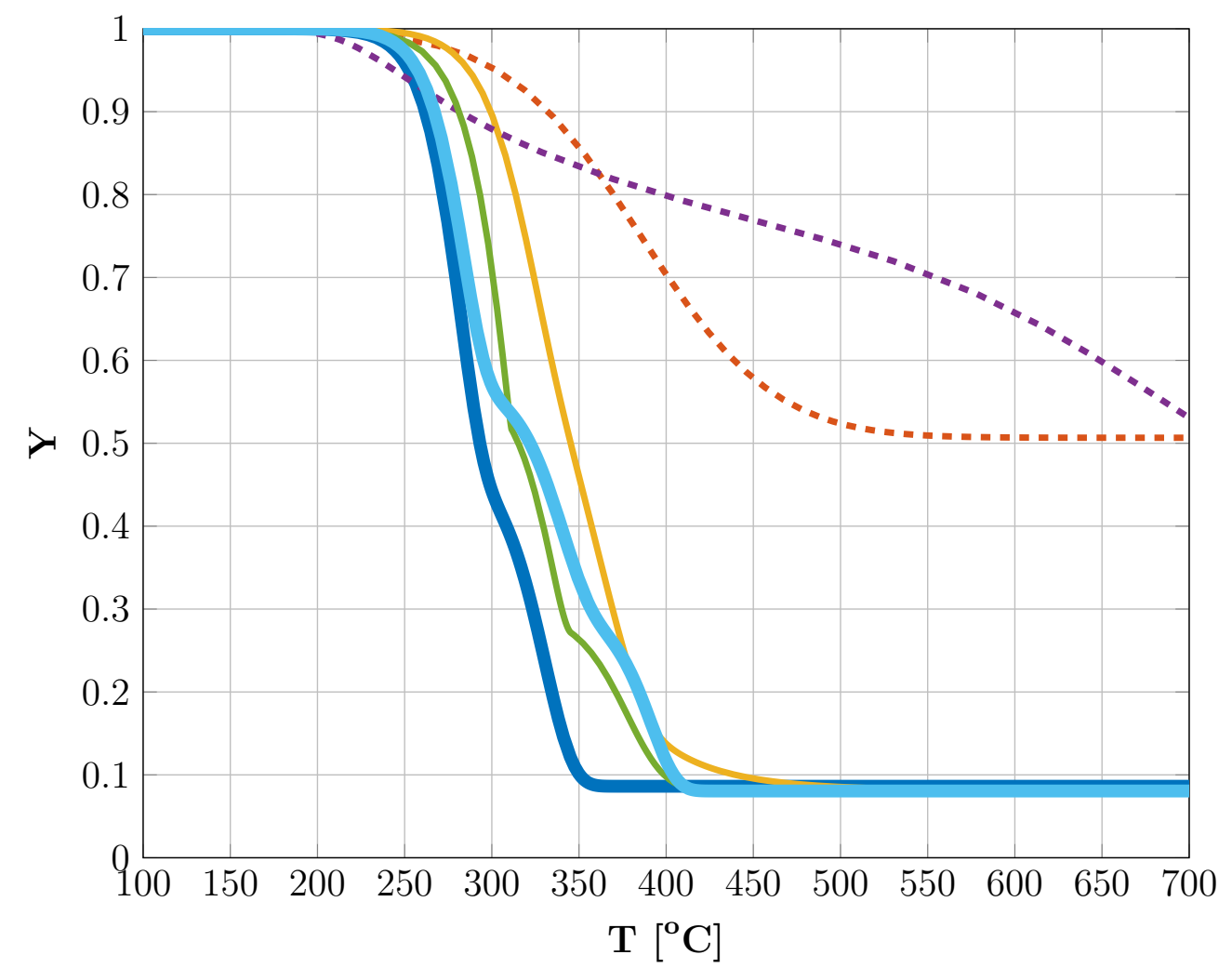

\begin{tabular}{|l}
$=$ Roberts et al.; $\beta=10$ \\
$=$ Chao \& Wang $2001 ; \beta=10$ \\
$=$ Rein et al. 2005; $\beta=10$ \\
$=$ Chao \& Wang $2001 ; \beta=20$ \\
- Rein et al. 2006; $\beta=20$ \\
Roberts et al.; $\beta=20$
\end{tabular}

Figure 5: Comparison of simulated TGA results using kinetic parameters from literature. PU, air. Solid lines: kinetic parameters from optimized models; dashed lines: kinetic parameters from graphical methods. This study is indicated with bold lines. $\beta\left[{ }^{\circ} \mathrm{C} / \mathrm{min}\right]$. 


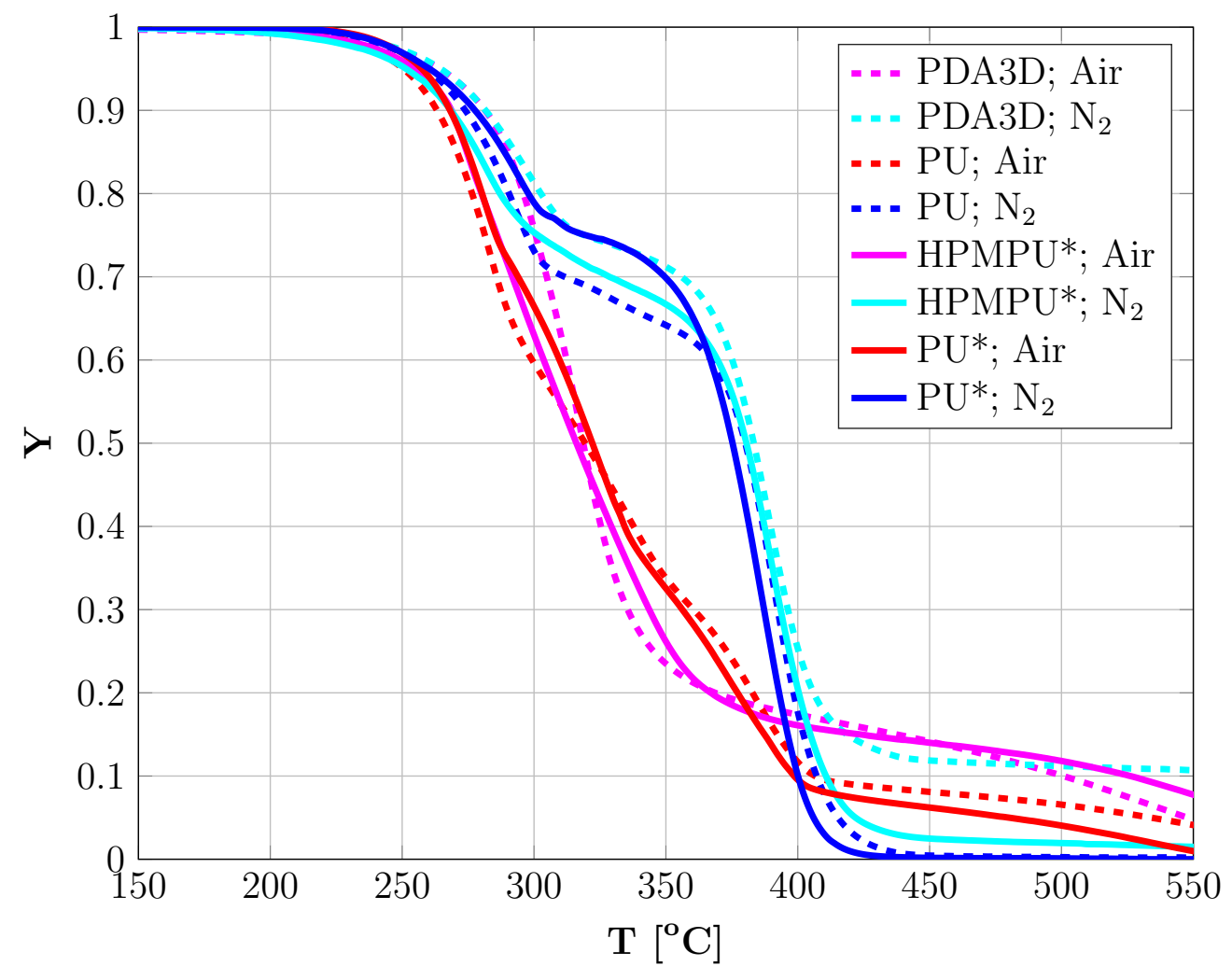

Figure 6: Comparison of neat PU, halophosphate-melamine flame retardant flexible polyurethane foam (HPMPU), and polydopamine coated flexible polyurethane foam (PDA3D) in air and $\mathrm{N}_{2}, \beta=20{ }^{\circ} \mathrm{C} / \mathrm{min} .{ }^{*}$ Data from Pau [26], Pau et al. [18]. 

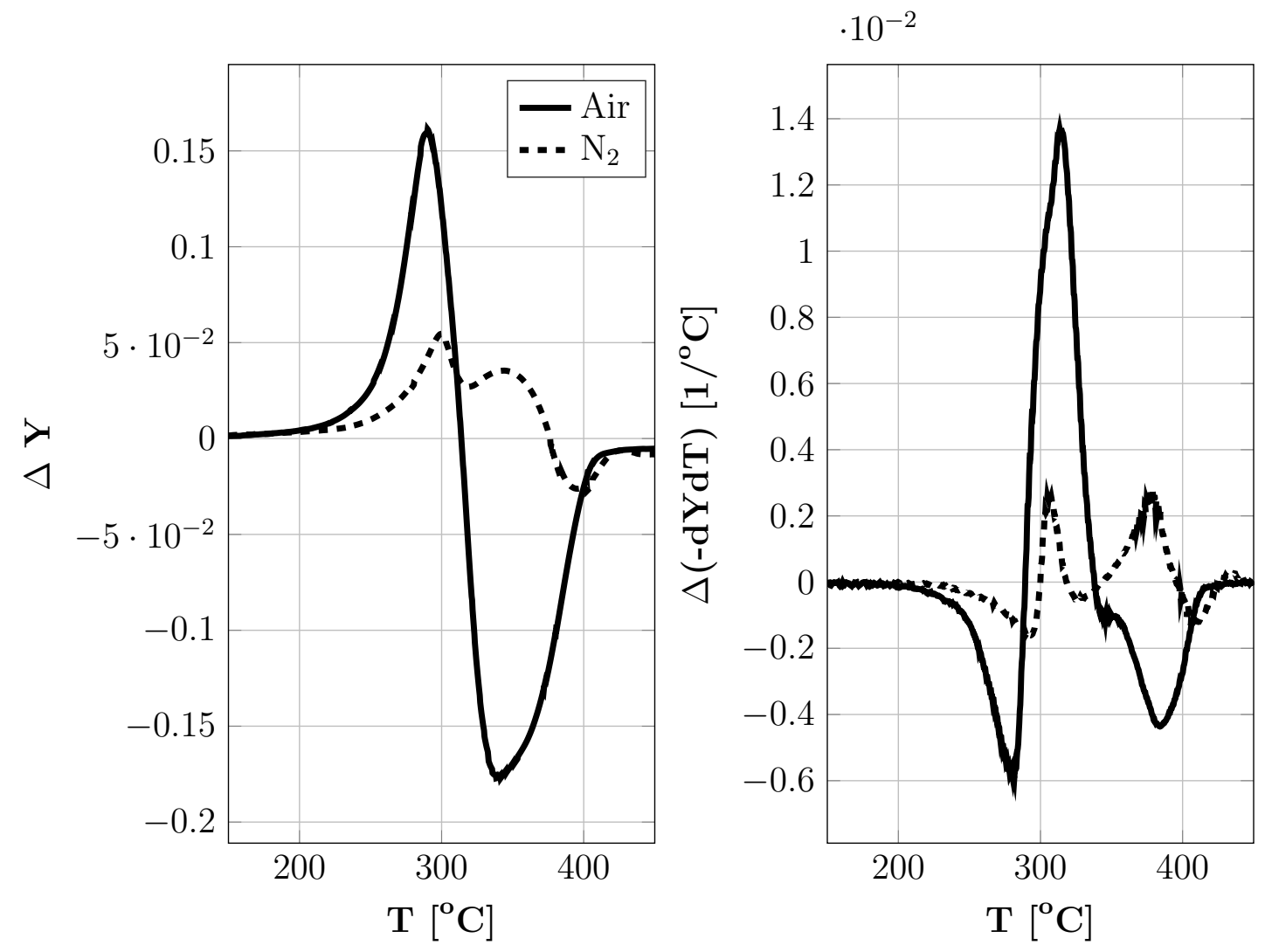

Figure 7: Difference between PDA3D and hypothetical (PDA+PU) in air and $\mathrm{N}_{2}, \beta=20$ ${ }^{\circ} \mathrm{C} /$ min. Differences of measured mass fraction and specific mass loss per ${ }^{\circ} \mathrm{C}$ vs. temperature. 